\title{
Investigating the Potential of Nigella Sativa and Thymoquinone in Salvaging the Embryo from Effects of Toxic Paternal Exposure to Cyclophosphamide
}

\author{
Suzanah Abdul Rahmana, Nadia Hanis Abdul Samat", Nur Amalina Ahmad", 'Afif Raihan Abdullah', Syazana \\ Mohamad Zahri ${ }^{\mathrm{a}}$, Saheera Kamarzaman ${ }^{\mathrm{b}}$

\begin{abstract}
aDepartment of Biomedical Science, Kulliyyah of Allied Health Sciences, International Islamic University Malaysia, Jalan Sultan Ahmad Shah, Bandar Indera Mahkota, 25200 Kuantan, Pahang.

${ }^{b}$ Anatomy Department, Faculty of Medicine, Cyberjaya University College of Medical Sciences, 3410, Jalan
\end{abstract} \\ Teknorat 3, Cyber 4, 63000, Cyberjaya, Selangor, Malaysia
}

\begin{abstract}
Background: Exposure to cyclophosphamide (CPA) for cancer treatment results in over-production of reactive oxygen species and oxidative stress thus affecting the DNA in male germ cell inducing sperm defects. Our goal is to assess the potential effects of Nigella sativa extract (NSE) and thymoquinone (TQ) on sperm and embryo quality following fertlization of sperm produced from germ cells which have been exposed to the damaging alkylating effects of CPA. Methods: Thirty six male ICR mice were divided into six groups; (I) Vehicle-treated control (normal saline), (II) CPA-only, (III) TQ-only, (IV) NSE-only, (V) CPA followed by TQ and (VI) CPA followed by NSE. Treatment with $200 \mathrm{mg} / \mathrm{kg}$ CPA and $10 \mathrm{mg} / \mathrm{kg}$ of both NSE and TQ were given by intraperitoneal injection. Animals were sacrificed at 33 days by cervical dislocation and sperm from caudal epidydymis were taken for analysis and in vitro fertilization (IVF) with eggs from untreated female. Fertilization rates and embryo development were monitored for 5 days. The result were analysed by using SPSS 16. Results: TQ and NSE supplementation to CPA-exposed male mice have no significant effect $(p>0.05)$ on the total number of sperm if compared to CPA-only exposed mice. NSE and TQ supplementation have been shown to have significant effect $(p<0.05)$ on the percentage of motile sperm as well as the number of abnormal sperm. Four types of abnormalities of the sperm were found which includes folded sperm, amorphous, banana-like and the head lacking of the usual hook. Finally, the embryo quality shows a significant improvement by the supplementation of TQ and NSE to CPA-exposed male mice $(p<0.05)$. Conclusion: Overall, both NSE and TQ have indicated chemopreventive potential against the cytotoxicity of cyclophosphamide on the reproductive capacity and fertility.
\end{abstract}

Keywords: Nigella sativa, thymoquinone, cyclophosphamide, in vitro fertilisation, mouse embryo.

\section{INTRODUCTION}

The majority of cancer survivors that received series of cancer treatment are still in their reproductive age. Chemotherapeutic drugs such as cyclophosphamide (CPA), commonly used to treat leukaemia, lymphomas and some types of brain cancer, has been reported to cause damage and interference with rapidly proliferating cells including germ cells. ${ }^{1}$ Chemotherapy drugs used has been proven to cause damage to germ cells in the testis possibly affecting the reproductive functions of cancer patients. The cyclophosphamide toxicity is

Corresponding author:

Assoc. Prof. Dr. Suzanah Abdul Rahman

Associate Professor

Department of Biomedical Science

Faculty of Allied Health Sciences

International Islamic University Malaysia

Jalan Sultan Ahmad Shah, Bandar Indera Mahkota

25200 Kuantan, Pahang, MALAYSIA

Tel: $+609-5705255$

Fax: $+609-5716776$

E-mail:arsuzanah@iium.edu.my induced by alkylation at N7 position of guanine and the formation of DNA-DNA cross-links, DNA-protein cross-links, and single-strand breaks. ${ }^{2}$ This affects the reproductive system by causing disturbance to the germ cells growth leading to premature menopause as well as sterility. CPA treatment causes significant systemic toxicity due to the overproduction of reactive oxygen species (ROS) leading to oxidative stress. ${ }^{3,4}$ In the males, the primary concern is that CPA can induce permanent azoospermia which may or may not be reversible in pre and post-pubertal patients. ${ }^{5}$ This study aims to assess the survival and damage of the embryo following fertilization with sperm of mice exposed to damaging alkylating effects of CPA.

Nigella sativa (NS), or Habbatussauda' is a known medicinal plant used in the Middle East since thousands of years before as a traditional medicine. Studies have discovered that thymoquinone (TQ), the active compound of NS essential oil, has contributed the most in the biological activity of this plant. ${ }^{4}$ The seeds have been reported to exhibit antioxidant ${ }^{6}$ and anti-inflammatory activity related 
to chemopreventive action. NS has also been reported to initiate the re-establishment of spermatogenesis following testicular injury. ${ }^{7}$ TQ has been proven to improve testicular histology appearance as well as reduce apoptotic activity prior to chronic toxic exposure to toluene. ${ }^{7}$ Similar results on the protective effects of NS on the cells in the testis from the effects of CPA in mice model were showed by Kamarzaman and colleagues. ${ }^{8}$

Assisted reproductive technology (ART) is now a widely accepted method in overcoming various obstacles to pregnancy and in treating problems of infertility. Assisted reproductive technology such as IVF has given hope to the patients to allow them to procreate and continue their generation. However, the advance technology also comes with disadvantages and imperfections that lead to the failure of the procedure. For instance, the unstable environment created for embryo to grow in vitro in IVF procedure produced weak embryo thus lead to implantation failure. The procedures of in vitro fertilization (IVF) and intracytoplasmic sperm injection (ICSI) have assisted couples experiencing infertility to achieve their dreams of parenthood.9 However, several factors such as injury to the gametes before fertilization, presence of free radicals, and the effects of the chemicals in the culture media may lead to the failure of the procedure. The integrity of sperm DNA can be predictive of the success of the procedure and may have impact on the development of the resulting embryo. ${ }^{10}$ The production of free radical-dependent fatty acids oxidation, on the other hand, can induce chromosomal breakage which can react with the embryo chromosome and lead to severe malformation. ${ }^{11,12} \mathrm{~A}$ study showed that $\mathrm{TQ}$, which is the active compound of NS, is a suitable exogenous antioxidant for preserving fair-quality embryos in vitro. $^{13}$

The effects of Nigella sativa extract (NSE) and its active compound, $T Q$, in the cultivation of the embryo as potential nurturing agents were investigated in this study. Non-invasive embryo examination was based on simple methods of observation focused on morphology and dynamics of embryo development under the inverted microscope without fixation and staining. A mechanistic understanding of the alteration to the normal embryo development following paternal chemical exposure and intervention with products of natural sources is the expected output of this study in the hope to provide an alternative solution to alleviate fertility setbacks towards an improved quality of life.

This study was conducted as an attempt to discover possibilities of NSE and its active compound, TQ, in improving sperm and embryo quality following toxic exposure to the alkylating chemotherapy drug, CPA. Effects of CPA which is believed to occur due to excessive production of ROS and affect the spermatogenesis, has the potential to be repaired by the presence of antioxidant. ${ }^{8,14,15}$

\section{MATERIALS \& METHODS}

\section{Animal}

Seventy-two ICR mice at 10-12 weeks (36 males and 36 females) were acclimatized for a week before starting the treatment. They were kept in polycarbonate cages inside a well-ventilated room with temperature $25^{\circ} \mathrm{C} \pm 3^{\circ} \mathrm{C}$, maintained under standard laboratory conditions; humidity ranged from $20 \%$ to $50 \%, 12$ hours light-dark cycle and were given water and food pallet ad libitum.

All animals were handled according to the criteria outlined in the Guidelines for Animal Study and approved by the Institutional Animal Care and Use Committee of the International Islamic University Malaysia (IACUC-IIUM) with the reference number IIUM/IACUC Approval/2013/(2)(5).

\section{Treatment Protocol}

A single dose of CPA was administered on day 1 by intraperitoneal injection (i.p.) at $200 \mathrm{mg} / \mathrm{kg}$ while NSE and TQ were continuously given also via i.p. with the dose of $10 \mathrm{mg} / \mathrm{kg}$ body weight on alternate days until day 32 . The male animals were treated based on the grouping as follows; Group 1-Vehicletreated control (normal saline); Group 2- CPA-only; Group 3- TQ-only; Group 4- NSE-only; Group 5- CPA followed by TQ (CPA-TQ) and Group 6- CPA followed by NSE (CPA-NSE). Day of treatment is designated as day 0 and the combination groups (group 5 and 6 ) were administered with CPA followed by NSE and TQ injection after 6 hours.

\section{Mouse Superovulation}

Each female mouse was injected with 20IU Gonal F (follicle stimulating hormone, Merck Serono) on day 31 for the stimulation of ovarian follicular growth. At 48 to 60 hours post-Gonal $F$ injection, the females received another injection of 30 IU hCG (human chorionic gonadotrophin) for ovulation.

\section{Sperm and Oocytes Collection}

All animals were euthanized on day 33 by cervical dislocation and both cauda epididymides were extracted and incubated at $37^{\circ} \mathrm{C}$ in sperm washing media for 30 minutes to allow them to swim-up. Sperm from cauda epididymis is used because the sperm in this area are matured and have high potential to fertilize oocytes. For fertilization and sperm analysis (sperm morphology, motility and count), sperm-media mixture was used.

Induced female mice were sacrificed with cervical dislocation and the oviduct was cut and kept inside IRVINE Multipurpose Handling Media (MHM). The oocytes were obtained from the oviduct by using a 
sterile needle under dissecting microscope in the form of cumulus oocyte complex (COCs). COCs were incubated inside the droplet of IRVINE complete culture media before fertilization.

\section{Sperm Morphology}

Ten micro litre of the sperm-media mixture was smeared on a glass slide and stained by haematoxylin and eosin staining. One hundred sperm were examined in order to classify them into abnormal or normal type of sperm. The abnormalities recorded can be categorised into four types; lack-hook (lack of sperm head hook), bananalike (the sperm head have banana-like form), amorphous (sperm with unclear shape head) and folded (sperm folded on themselves). ${ }^{16}$

\section{Sperm Motility}

Ten micro litre of the sperm-media mixture was mixed with $90 \mu \mathrm{l}$ tap water on a pre-warmed glass slide and covered with a cover slip. The percentage of motile sperm (\%) was estimated by assessing the slide using phase contrast microscope.

\section{Sperm Count}

Total number of sperm/ml was calculated using haemocytometer. Ten $\mu \mathrm{l}$ sperm-media mixture was suspended in $90 \mu \mathrm{l}$ tap water by using a pipette to create a ten times dilution. Then, $10 \mu \mathrm{l}$ from the sperm water mixture was placed into the counting chamber on haemocytometer and covered with cover slip. Sperm concentration (sperm count) in every $\mathrm{ml}$ was later counted by using the formula as below;

Concentration of sperm $=$ Number of sperm in five squares $X 10$ (dilution) X 5 squares $X 10000$.

\section{DNA Integrity Assessment}

Ten micro litre of the sperm-media mixture was smeared on glass slide and stained with acridine orange. The slides were examined using a fluorescence microscope under 40X magnification with $490 \mathrm{~nm}$ excitation light and $530 \mathrm{~nm}$ barrier filter. Stained sperm (head) appeared as green, yellow or red in colour. Green stained sperm indicates presence of double-stranded DNA while yellow or red indicates single-stranded DNA ${ }^{17}$. One hundred spermatozoa from each slide were counted in order to get the percentage of double-stranded DNA.The percentage of double-stranded DNA from each sample was calculated by using the formula below;

Number of green stained sperm $\times 100$

Total number of stained sperm (red,yellow ,green)

\section{In vitro fertilization}

The amount of sperm needed for insemination was aliquoted from sperm and washing media mixture and placed into $50 \mu \mathrm{l}$ droplet of complete culture media containing COCs. The cultures were checked for any sign of fertilization, after 2 hours post insemination. Fertilized oocytes were then transferred into new droplets of culture media and embryo development was assessed after 24 hours.

\section{Embryo evaluation and grading}

All embryos were evaluated and scored based on the equality of blastomeres size as well as percentage of fragmentation according to that by Baczkowski, Kurzawa, \& Głabowski, $2004{ }^{18}$.

\section{Statistical analysis}

Statistical evaluations were performed using Oneway ANOVA parametric test followed by Tukey test as the post-hoc test. SPSS 16.0 software was used for statistical analysis and $p$-value $<0.05$ was considered as significant.

\section{RESULTS}

\section{Sperm count}

Table I presents the results for sperm analysis including sperm count, motility, morphology and DNA fragmentation analysis. It was found that there is no significant difference in sperm count between the groups. However, descriptively, the NSE alone group seemed to possess a higher number of sperm count. CPA-NSE and CPA-TQ groups showed higher sperm count than the CPA group.

\section{Sperm motility}

CPA group showed significant reduction of percentage of motile sperm $(p$-value $<0.05)$ when compared to the control group (Table 1). On the other hand, NSE and TQ group show a significant increment compared to CPA group ( $p$-value $<0.05)$. The CPA-NSE and CPA-TQ groups have also shown a significant increment in sperm motility (\%) if compared to CPA ( $p$-value $<0.05)$. However, there is no significant difference between the groups.

\section{Sperm morphology- Number of abnormal sperm}

CPA group showed no significant difference between the groups. A significant high number of abnormal sperm as compared to the control group ( $p$-value $<0.05$ ) was observed as shown in Table 1 . NSE and TQ group shows the lowest number of abnormalshaped sperm as compared to the CPA group $(p$-value $=0.0001)$. In addition, both CPA-NSE and CPA-TQ displayed a lower number of abnormal sperm as compared to CPA group ( $p$-value $<0.05)$. 


\section{DNA fragmentation}

DNA integrity assessment showed no significant difference between all groups. However, descriptively, CPA group showed higher number of single stranded DNA and supplementation with NSE and TQ, either alone or after CPA exposure showed a decrement of single stand DNA presence when compared to the CPA group (Table I).

Table I Sperm analysis

\begin{tabular}{|c|c|c|c|c|c|c|}
\hline & CONTROL & CPA & NSE & $\mathrm{TQ}$ & CPA NSE & CPA TQ \\
\hline $\begin{array}{l}\text { Sperm } \\
\text { Count } \\
10^{7} / \mathrm{ml}\end{array}$ & $13.3 \pm 9.9$ & $8.46 \pm 5.38$ & $23.1 \pm 13.68$ & $20.3 \pm 13.68$ & $9.51 \pm 3.42$ & $18.20 \pm 10.92$ \\
\hline $\begin{array}{l}\text { Sperm } \\
\text { Motility (\%) }\end{array}$ & $76.67 \pm 8.16$ & $48.00 \pm 14.83^{*}$ & $73.75 \pm 14.08^{\dagger}$ & $77.50 \pm 8.86^{\dagger}$ & $70.00 \pm 7.56^{\dagger}$ & $78.00 \pm 8.37^{\dagger}$ \\
\hline $\begin{array}{l}\text { No. of } \\
\text { Abnormal } \\
\text { Sperm }\end{array}$ & $33.67 \pm 4.32$ & $58.8 \pm 8.08^{*}$ & $32.62 \pm 8.96^{\dagger}$ & $33.25 \pm 5.78^{\dagger}$ & $40.75 \pm 7.25^{\dagger}$ & $38.00 \pm 2.65^{\dagger}$ \\
\hline $\begin{array}{l}\text { No. of } \\
\text { Single } \\
\text { Stranded } \\
\text { DNA }\end{array}$ & $49.5 \pm 15.16$ & $62.4 \pm 17.62$ & $39.75 \pm 12.36$ & $42.12 \pm 15.38$ & $39.38 \pm 12.84$ & $39.40 \pm 8.02$ \\
\hline
\end{tabular}

The values are expressed as mean \pm S.D

*Significantly different from control group, $p<0.05$.

†Significantly different from CPA group, $p<0.05$.

\section{Types of Abnormalities}

Four common abnormalities detected were lack of the usual hook (LH), banana-like form head (BL), amorphous (A) and folded $(F)$. From the data, we can conclude that the most common abnormality in the sperm head morphology is folded, followed by amorphous, banana like and lack of usual hook (Table II). The number of LH type of abnormal sperm were found to be statistically significant lower in NSE, TQ and CPA-TQ as compared to CPA group.
However, there is no significant difference between the groups.

\section{Fertilization Rate}

Treatment with NSE showed a significant reduction on the fertilization rate after IVF ( $p$-value $<0.05)$. Other groups showed no significant difference in comparison with control and CPA group as in Table III.

Table II Sperm abnormalities

\begin{tabular}{lllllll}
\hline Types & Control & CPA & NSE & TQ & CPA-NSE & CPA-TQ \\
\hline LH & $3.83 \pm 3.06$ & $10.6 \pm 1.95$ & $1.88 \pm 2.36^{\dagger}$ & $1.38 \pm 1.51^{\dagger}$ & $6 \pm 4.11$ & $3.2 \pm 2.86^{\dagger}$ \\
BL & $5.17 \pm 1.72$ & $9.4 \pm 4.04$ & $6.62 \pm 3.46$ & $8.38 \pm 3.93$ & $5.62 \pm 4.47$ & $9.2 \pm 2.86$ \\
A & $8.17 \pm 4.26$ & $10.6 \pm 4.04$ & $10.75 \pm 3.28$ & $8.12 \pm 4.42$ & $10 \pm 6.02$ & $10.8 \pm 4.15$ \\
F & $16.5 \pm 3.72$ & $28.2 \pm 6.83$ & $13.38 \pm 5.13^{\dagger}$ & $15.38 \pm 2.97^{\dagger}$ & $19.12 \pm 3.44^{\dagger}$ & $14.8 \pm 5.2^{\dagger}$ \\
\hline
\end{tabular}

The values are expressed as mean \pm S.D

${ }^{\dagger}$ Significantly different from CPA group, $p<0.05$.

Table III The fertilization rate after IVF.

\begin{tabular}{lcccccc}
\hline & CONTROL & CPA & NSE & TQ & CPA NSE & CPA TQ \\
\hline NO OF OOCYTES & 243 & 49 & 228 & 297 & 84 & 47 \\
FERTILIZED OOCYTES & 180 & 25 & 40 & 167 & 50 & 29 \\
FERTILIZATION RATE (\%) & 74.1 & 51.0 & $17.5^{*}$ & 56.2 & 59.5 & 61.7 \\
\hline
\end{tabular}

The values are expressed as mean \pm S.D

*Significantly different from Control group, $p<0.05$. 


\section{Embryo fragmentation}

The embryo has been graded based on fragmentation formed (grade I, II, III and IV) and the structure of blastomere (type A, B and C). ${ }^{18}$ NSE group showed statistically significant higher number of grade I embryo if compared to control group ( $p$ value $<0.05$ ) as shown in Table IV. Moreover, CPANSE and CPA-TQ show statistically significant reduction in grade I embryo if compared to control group ( $p$-value $<0.05$ ). As for embryos of grade II type, CPA group showed statistically significant low number of embryo grade II as compared to control group with $p$-value $<0.05$. The TQ group also showed significant difference in comparison with CPA group. Furthermore, for grade III, there is no significant difference between the groups while for grade IV the statistic is not available as no grade IV embryo was produced during the IVF.

\section{The structure of blastomeres}

There is no significant difference in the structure of blastomeres between groups for the number of type A embryo (Table V). However, the percentage of type B blastomeres from group CPA, NSE, CPA NSE and CPA TQ were significant lower than control group. While TQ group showed significantly higher in the number of type $B$ embryo as compared with CPA group ( $p$-value $<0.05$ ). Lastly, based on embryo grading, there was no significant different in the percentage of type $\mathrm{C}$ mice blastomeres between the groups.

Table IV The number and percentage of embryos presenting fragmentation grades following 24-hours of in vitro culture

\begin{tabular}{lllllll}
\hline & CONTROL & CPA & NSE & TQ & CPA-NSE & CPA-TQ \\
\hline Grade I & $32(17.8 \%)$ & $6(24.0 \%)$ & $5(12.5 \%)^{*}$ & $25(15.0 \%)$ & $10(20.0 \%)$ & $6(20.7 \%)$ \\
Grade II & $131(72.8 \%)$ & $8(32.0 \%)^{*}$ & $32(80.0 \%)^{*}$ & $110(65.9 \%)^{\dagger}$ & $37(74.0 \%)^{*}$ & $20(69.0 \%)^{*}$ \\
Grade III & $17(9.4 \%)$ & $11(44.0 \%)$ & $3(7.5 \%)$ & $32(19.2 \%)$ & $3(6.0 \%)$ & $3(10.3 \%)$ \\
Grade IV & $0(0 \%)$ & $0(0 \%)$ & $0(0 \%)$ & $0(0 \%)$ & $0(0 \%)$ & $0(0 \%)$ \\
\hline Total & $180(100 \%)$ & $25(100 \%)$ & $40(100 \%)$ & $167(100 \%)$ & $50(100 \%)$ & $29(100 \%)$
\end{tabular}

Data is presented in number and \% of embryos

*Significantly different from control group, $p$-value $<0.05$.

†Significantly different from CPA group, $p$-value $<0.05$.

Table V The number and percentage of embroys presenting the structure of mice blastomeres following 24 hours in-vitro culture

\begin{tabular}{lllllll}
\hline Group & CONTROL & CPA & NSE & TQ & CPA NSE & CPA TQ \\
\hline Type A & $35(19.4 \%)$ & $3(12.0 \%)$ & $6(15.0 \%)$ & $11(6.6 \%)$ & $22(44.0 \%)$ & $18(62.1 \%)$ \\
Type B & $144(80.0 \%)$ & $17(68.0 \%)^{*}$ & $29(72.5 \%)^{*}$ & $154(92.2 \%)^{\dagger}$ & $28(56.0 \%)^{*}$ & $10(34.5 \%)^{*}$ \\
Type C & $1(0.6 \%)$ & $5(20.0 \%)$ & $5(12.5 \%)$ & $2(1.2 \%)$ & $0(0.0 \%)$ & $1(3.4 \%)$ \\
Total & $180(100 \%)$ & $25(100 \%)$ & $40(100 \%)$ & $167(100 \%)$ & $50(100 \%)$ & $29(100 \%)$ \\
Total & $180(100 \%)$ & $25(100 \%)$ & $40(100 \%)$ & $167(100 \%)$ & $50(100 \%)$ & $29(100 \%)$ \\
\hline
\end{tabular}

Data is presented in percentage

*Significantly different from control group, $\mathrm{p}<0.05$

†'Significantly different from CPA group, $p<0.05$

\section{DISCUSSION}

Interruptions or problem during spermatogesesis can be detected by determining several markers such as the concentration of sperm produced. Damage to rapidly proliferating cells may lead to a decrease in the number of sperm produced, classified as azoospermia. Sperm count is one of the important parameters used to detect any abnormalities in the male reproductive system. ${ }^{19}$ However, in this study, no significant differences were seen in the count between the groups. A small increment in the concentration of sperm was observed in CPA-NSE and CPA-TQ group as compared to CPA. This could indicate that post treatment with either NSE or TQ after CPA exposure has the potential of inducing DNA repair thus leading to an improvement by increasing the concentration of sperm produced. Another study proposed that antioxidants from NSE and TQ have the ability to reduce the production of ROS in cell culture media as well as protect living cells from the dangerous effects exerted by ROS. ${ }^{20}$ Thus, the improvement discovered may indicate that NSE and TQ possess curative effects by functioning as antioxidants which facilitate in creating oxidant and pro-oxidant balance as well as scavenge the free radical produced. ${ }^{21}$

A reduction in sperm motility and movement are among the important signal of male infertility. ${ }^{22}$ Mitochondrial damage in the sperm head is believed to be one of the causes of reduction in sperm motility $^{23}$ as mitochondria play an important role in supplying energy to the sperm to function normally. Adenosine triphosphate (ATP) is the common form of energy used for cellular metabolism. Its generation process takes place in the cytoplasm or mitochondria. ${ }^{24}$ Sperm motility is facilitated when microtubules use the energy that is transferred from the mitochondrial inner membrane. A stable and 
continuous supply of energy is a crucial requirement for allowing excellent cellular function. At the optimum motility stage of a spermatozoon, more energy is required. ${ }^{25}$ The results on sperm motility showed that the CPA group possesses the least percentage of motile sperm. However, NSE and TQ group have statistically significant increment in motility percentage as compared to CPA. Likewise, CPA-NSE and CPA-TQ group also show statistically significant increment in motility. These results show that treatment with either NSE or TQ post CPA administration prevent mitochondrial damage thus improve sperm motility. The presence of ROS in the sperm cell due to CPA exposure with reduced antioxidant activity may caused modification on genetic material thus affecting the mitochondria performance and resulting the decrement of sperm motility. Action of reactive oxygen species on spermatozoa resulted in decreased capacity for ionophore-induced acrosome reaction and become the reason of sperm motility decrement. ${ }^{2}$

The morphology of the sperm is another important indication of sperm quality. A good mouse sperm has a hook-shaped head with a single line tail. The increment of sperm head abnormalities in mice following CPA exposure indicates that the active metabolite of the alkylating drug may have affected the germ cells and caused damage to the DNA and lead to the production of abnormal sperm. DNA synthesis will be interrupted since the process occurs before pre-meiotic phase and no further DNA synthesis occurs throughout spermatogenesis in the cell cycle. ${ }^{26,27}$ In addition, exposure to chemical mutagens could cause abnormalities in seminal fluid resulting in functional or structural impairment of sperm and significantly increasing the frequency of mistakes in the spermatozoon differentiating process during spermatogenesis. ${ }^{28}$ The occurrence of sperm head abnormalities have also been attributed to the occurrence of chromosomal aberration mutations to testicular DNA that occur during the packaging of genetic material in the sperm head. ${ }^{16}$ In the present study, it was found that there has been a significant increase in the number of abnormal sperm morphology, from 34\% (control) to $59 \%$ in CPA group. Administrations of NSE following treatment with CPA resulted in a statistically significant decrease in the percentage of total sperm head abnormalities. Previous study has reported that carcinogenic chemicals like CPA induced sperm abnormalities up to $1.2 \%$ to $3.4 \%$ in comparison to control values. ${ }^{16}$ Tripathi and Jena ${ }^{29}$ similarly reported that at $100 \mathrm{mg} / \mathrm{kg}$ and $200 \mathrm{mg} / \mathrm{kg}$ doses, CPA significantly enhanced the abnormality in sperm head morphology.

The results of this study also showed that NSE and TQ groups have significant lower number of abnormal sperm if compared to CPA group. CPA-NSE and CPA-TQ also showed lower number of abnormal sperm as compared to CPA group. This indicates that NSE and TQ have the potential to prevent the production of abnormal sperm. Previous studies have designated NS and its active metabolite, TQ as efficient antioxidant quenchers. ${ }^{30}$ The cause of the chemoprotective effect of NS might be due to the presence of biological active compounds that mainly comprise of antioxidants. TQ and NS inhibit generation of ROS by protection against lipid peroxidation $^{30}$ and detoxification of cytotoxic radicals was also suspected to contribute to DNA repair. TQ has been proven to have antiinflammatory properties and is known to have chemoprotective action. ${ }^{31}$ In our earlier study, we found that TQ protected testis and spermatozoa from toxicity and DNA fragmentation induced by CPA. ${ }^{8}$

Sperm DNA integrity also plays an important role for the normal development of the embryo, foetus and new-borns. ${ }^{17,32}$ It is also stated that the integrity of the sperm is crucial in fertilization as it involves sperm capacitation and acrosome reaction. ${ }^{2,33}$ The male gamete's genetic integrity should be in the best state in order to have a successful and healthy pregnancy. ${ }^{33}$ However, many authors reported that the spermatozoa with fragmented DNA are also able to fertilize an egg and the DNA damage does not influence the rate of fertilization. ${ }^{2,10}$ The DNA damage caused by CPA can affect mitochondrial and nuclear DNA. ${ }^{17}$ DNA damage can happen during spermatogenesis when the oxygen radical released by the alkylating agent make the process happened under oxidative-stressed condition. ${ }^{3,34}$ The results from this study indicate that there is no statistically significant difference in terms of DNA integrity between the test groups. However, descriptively, CPA group showed highest number of single stranded DNA which appear as red under fluorescence microscope prior to acridine orange staining. This can be supported by previous study which discovered that CPA induce injury to the testicular tissue, cause the morphological alteration and lead to DNA fragmentation.

Damage caused by CPA on sperm also affects the ability of the sperm to penetrate and fertilize the oocyte. The ability of sperm to fertilize the oocytes depends on several factors such as the morphology of the sperm head and motility. Defects in these factors will lead to a low percentage of fertilization. The result showed that NSE group exhibited a statistically significant decrement of number of fertilized oocyte as compared to control. Reasons for this reduction have been investigated carefully as conceptually, the NSE dosage should not cause negative effect on fertilization rate. However, it can be related to the crowded environment in the culture media due to high number of embryo in each culture media droplet. Furthermore, descriptively, CPA-NSE and CPA-TQ showed slightly higher percentages of fertilized egg as compared to the CPA group. This could mean that either NSE or TQ has chemopreventive effect against sperm degradation. Previous finding claims 
the histopathological and morphometrical changes induced by CPA in the testis can be improved by TQ administration 35 as well as protecting rapidly dividing spermatogonia from the alkylating effects of CPA. Thus, the improvements in the structure and spermatogenesis explained the increase in fertilization rate.

Embryo quality produced by IVF can be influenced by several factors including environment of the culture as well as genetic information transferred from both parents. Male infertility due to the damages occur in the genetic material which might lead to the poor quality embryo. The embryo can be classified based on percentage of fragments formed inside the embryo and the structure of the blastomere. According to previous study, the limitations towards implantation will be increased proportionally to the percentage of fragmentations formed in the embryo. ${ }^{36}$ Based on results, it is found that supplementation of NSE and TQ after CPA exposure of the male mice produced a higher number of grade II and type B embryo. Similarly, CPA-NSE and CPA-TQ groups showed statistically significant increase in the number of grade II embryo. While in terms of the structure of blastomeres, CPA-NSE and CPA-TQ showed an increment in the number of type $B$ embryo for both groups if compared to the CPA group, descriptively. Therefore, the increment indicates that there is potential of repair and prevention from sperm damage caused by the alkylating agent, CPA, with the use of NSE and TQ may improve the quality of embryo produced.

\section{CONCLUSIONS}

Collectively, the findings in this study may promote the potential of NSE and TQ as a supplementation following paternal exposure to CPA thus signifying the beneficial effects of the extract and its active ingredient in the protection and prevention of the male gametes from cellular and structural damage. The results also indicate the potential of NSE and TQ in the reduction of toxic effects of chemotherapeutic agents as well as in improving the quality of life of the patients following treatment.

\section{ACKNOWLEDGEMENT}

The authors would like to thank the Ministry of Higher Education of Malaysia for funds under the Exploratory Research Grant Scheme (ERGS-13-0130046).

\section{REFERENCES}

1. Codrington $A M$, Hales BF, Robaire B. Exposure of male rats to cyclophosphamide alters the chromatin structure and basic proteome in spermatozoa. Human Reproduction. 2007;22 (5):1431-42.
2. Elangovan N, Chiou TJ, Tzeng WF, Chu ST. Cyclophosphamide treatment causes impairment of sperm and its fertilizing ability in mice. Toxicology. 2006;222(1-2):60-70.

3. Mitchell DC, Niu S-L, Litman BJ. Enhancement of $\mathrm{G}$ protein-coupled signaling by DHA phospholipids. Lipids. 2003;38(4):437-43.

4. Alenzi FQ, El-Bolkiny YE-S, Salem ML. Protective effects of Nigella sativa oil and thymoquinone against toxicity induced by the anticancer drug cyclophosphamide. Br J Biomed Sci. 2010;67 (1):20.

5. Meistrich ML. Male gonadal toxicity. Pediatr Blood Cancer. 2009;53(2):261-6.

6. Badary OA, Taha RA, Gamal El-Din AM, AbdelWahab MH. Thymoquinone is a potent superoxide anion scavenger. Drug Chem Toxicol. 2003;26(2):87-98.

7. Kanter M. Thymoquinone attenuates lung injury induced by chronic toluene exposure in rats. Toxicol Ind Health. 2011;27(5):387-95.

8. Kamarzaman S, Sha M, Rahman SA. Effects on Mouse Spermatogenesis and DNA Fragmentation Following Exposure to Cyclophosphamide and Thymoquinone. European International Journal of Science and Technology. 2013;2(7):119-36.

9. Verhaak, Chris M., et al. "Women's emotional adjustment to IVF: a systematic review of 25 years of research." Human reproduction update. 2007; 27-36.

10. Morris ID, llott S, Dixon L, Brison DR. The spectrum of DNA damage in human sperm assessed by single cell gel electrophoresis (Comet assay) and its relationship to fertilization and embryo development. Hum Reprod. 2002;17(4):990-8.

11. Emerit I. Reactive oxygen species, chromosome mutation, and cancer: possible role of clastogenic factors in carcinogenesis. Free Radic Biol Med. 1994;16(1):99-109.

12. Alhimaidi A. Thymoqunone treatment of intracytoplasmic sperm injection (ICSI) compared to in vitro fertilization (IVF) of mice oocytes and their development in vitro. Zoology. J Adv Mol Med [Internet]. 2005; Available from: http://www.advmolmed.com/issue/20053/ pdf/04.pdf

13. Kamarzaman S, Yazmie A, Wahab A, Rahman SA. Effects of Thymoquinone Supplementation on Cyclophosphamide Toxicity of Mouse Embryo In vitro. Global Veterinaria. 2014;12(1):80-90.

14. Rezvanfar M, Sadrkhanlou R, Ahmadi a, ShojaeiSadee H, Mohammadirad a, Salehnia a, et al. Protection of cyclophosphamide-induced toxicity in reproductive tract histology, sperm characteristics, and DNA damage by an herbal source; evidence for role of free-radical toxic stress. Hum Exp Toxicol [Internet]. 2008 Dec [cited 2014 Mar 9];27(12):901-10. Available from: http://www.ncbi.nlm.nih.gov/ pubmed/19273545

15. Chabra a, Shokrzadeh M, Naghshvar F, Salehi F, Ahmadi a. Melatonin ameliorates oxidative 
stress and reproductive toxicity induced by cyclophosphamide in male mice. Hum Exp Toxicol [Internet]. 2014;33(2):185-95. Available from: http://www.ncbi.nlm.nih.gov/ pubmed/23703819

16. Wyrobek AJ, Eskenazi B, Young S, Arnheim N, Tiemann-Boege I, Jabs EW, et al. Advancing age has differential effects on DNA damage, chromatin integrity, gene mutations, and aneuploidies in sperm. Proc Natl Acad Sci. 2006;103(25):9601-6.

17. Soleimanzadeh A, Saberivand A. Effect of curcumin on rat sperm morphology after the freeze-thawing process. Veterinary Research Forum. 2013;4(3):185-9.

18. Baczkowski T, Kurzawa R, Głabowski W. Methods of embryo scoring in in vitro fertilization. Reprod Biol. 2004;4(1):5-22.

19. Drumond AL, Weng CC, Wang G, Chiarini-Garcia $H$, Eras-Garcia L, Meistrich ML. Effects of multiple doses of cyclophosphamide on mouse testes: accessing the germ cells lost, and the functional damage of stem cells. Reprod Toxicol [Internet]. 2011 Dec [cited 2014 Aug 11];32 (4):395-406. Available from: http:// www.pubmedcentral.nih.gov/ articlerender.fcgi?artid=3236636\&tool= pmcentrez\&rendertype=abstract

20. Mousavi SH, Tayarani-Najaran Z, Asghari M, Sadeghnia HR. Protective effect of Nigella sativa extract and thymoquinone on serum/ glucose deprivation-induced PC12 cells death. Cell Mol Neurobiol [Internet]. 2010 May [cited 2014 Mar 19];30(4):591-8. Available from: http://www.ncbi.nlm.nih.gov/ pubmed/20054635

21. Ismail M, Al-Naqeep G, Chan KW. Nigella sativa thymoquinone-rich fraction greatly improves plasma antioxidant capacity and expression of antioxidant genes in hypercholesterolemic rats. Free Radic Biol Med. 2010;48(5):664-72.

22. Holt W V, Penfold LM, Chenoweth P, Lorton S, others. Fundamental and practical aspects of semen cryopreservation. Anim Androl Theor Appl. 2014;76.

23. Jarrell JF, Bodo L, Younglai E V, Barr RD, O'Connell GJ. The short-term reproductive toxicity of cyclophosphamide in the female rat. Reprod Toxicol. 1991;5(6):481-5.

24. Amaral A, Lourenço B, Marques M, RamalhoSantos J. Mitochondria functionality and sperm quality. Reproduction. 2013;146(5).

25. Lemma A. Effect of cryopreservation on sperm quality and fertility. INTECH Open Access Publisher; 2011.

26. Otubanjo OA, Mosuro AA. An in vivo evaluation of induction of abnormal sperm morphology by some anthelmintic drugs in mice. Mutat Res Toxicol Environ Mutagen. 2001;497(1):131-8.

27. Odeigah PGC. Sperm head abnormalities and dominant lethal effects of formaldehyde in albino rats. Mutat Res Toxicol Environ Mutagen. 1997;389(2):141-8.
28. Bakare AA, Mosuro AA, Osibanjo O. An in vivo evaluation of induction of abnormal sperm morphology in mice by landfill leachates. Mutat Res Toxicol Environ Mutagen. 2005;582(1):2834.

29. Tripathi DN, Jena GB. Astaxanthin inhibits cytotoxic and genotoxic effects of cyclophosphamide in mice germ cells. Toxicology. 2008;248(2):96-103.

30. Taylor A, Robson A, Houghton BC, Jepson CA, Ford WCL, Frayne J. Epididymal specific, selenium-independent GPX5 protects cells from oxidative stress-induced lipid peroxidation and DNA mutation. Hum Reprod. 2013;28(9):233242.

31. Sethi G, Ahn KS, Aggarwal BB. Targeting nuclear factor-kappa B activation pathway by thymoquinone: role in suppression of antiapoptotic gene products and enhancement of apoptosis. Mol Cancer Res. 2008;6(6):105970.

32. Varghese a C, Bragais FM, Mukhopadhyay D, Kundu S, Pal M, Bhattacharyya a K, et al. Human sperm DNA integrity in normal and abnormal semen samples and its correlation with sperm characteristics. Andrologia. 2009 Aug;41(4):207-15.

33. Meseguer M, Santiso R, Garrido N, GarcíaHerrero S, Remohí J, Fernandez JL. Effect of sperm DNA fragmentation on pregnancy outcome depends on oocyte quality. Fertil Steril. 2011 Jan;95(1):124-8.

34. Sakkas D, Shoukir Y, Chardonnens D, Bianchi PG, Campana a. Early cleavage of human embryos to the two-cell stage after intracytoplasmic sperm injection as an indicator of embryo viability. Hum Reprod [Internet]. 1998 Jan;13(1):182-7. Available from: http://www.ncbi.nlm.nih.gov/ pubmed/9512254

35. Kamarzaman S, Shaban M, Rahman S. The prophylactic effect of Nigella sativa against cyclophosphamide in the ovarian follicles of matured adult mice: a preliminary study. JAPS, J Anim ... [Internet]. 2014 [cited 2014 Mar 19];24(1):81-8. Available from: http:// www.thejaps.org.pk/docs/v-24-1/13.pdf

36. Erenus M, Zouves C, Rajamahendran P, Leung S, Fluker M, Gomel V. The effect of embryo quality on subsequent pregnancy rates after in vitro fertilization. Fertil Steril. 1991;56(4):70710. 